\title{
La tombe monumentale de Grotte Scalina (Viterbe)
}

Campagne de fouilles 2016

Vincent Jolivet et Edwige Lovergne

\section{(2) OpenEdition \\ Journals}

Édition électronique

URL : http://journals.openedition.org/cefr/1653

DOI : $10.4000 /$ cefr. 1653

ISSN : 2282-5703

Éditeur

École française de Rome

Référence électronique

Vincent Jolivet et Edwige Lovergne, « La tombe monumentale de Grotte Scalina (Viterbe) », Chronique des activités archéologiques de l'École française de Rome [En ligne], Italie centrale, mis en ligne le 24 janvier 2017, consulté le 15 novembre 2019. URL : http://journals.openedition.org/cefr/1653 ; DOI: 10.4000/cefr. 1653

Ce document a été généré automatiquement le 15 novembre 2019.

(c) École française de Rome 


\section{La tombe monumentale de Grotte Scalina (Viterbe)}

Campagne de fouilles 2016

Vincent Jolivet et Edwige Lovergne

\section{NOTE DE L'AUTEUR}

Ont participé aux travaux de la campagne 2016 : Maxime Brugellis (Université de Paris 1-Panthéon Sorbonne), Aristide Chryssoulis (Université de Paris 1-Panthéon Sorbonne), Niccolò Corti (Università di Firenze), Anaïs Daumont-Marx (Paris), Niccolò Daviddi (Università di Firenze), Marie De Jonghe (Université de Paris 1-Panthéon Sorbonne), Henri de Megille (Université de Paris 1-Panthéon Sorbonne), Manuel De Souza (Université de Saint-Étienne), Saddam Douadi (Université Hassiba Benbouadi, Chlef), Anna Duday (Bordeaux), Baptiste Duvert (Université de Tours), Karine Gillet (CNRS, UMR 8546, Paris), Lou Godefroy (Université de Paris 1-Panthéon Sorbonne), Martin Jaillet (École normale supérieure, Paris), Anders Joelsson (Stockholm), Andy Kellog (Université de Tours), Julie Labregère (Université de Tours), Lucie Motta (Université de Lyon), Pascal Neaud (INRAP, Reims), Luca Pesante (Roma), Birgita Rask (Stockholm), Emma Vallet (Lyon) e Caroline Vanderberghe (Université de Paris 10).

1 La sixième campagne de fouilles sur le site de la tombe monumentale de Grotte Scalina, placée sous le régime de la concession octroyée par le Ministero dei Beni e delle Attività culturali e del turismo à l'École normale supérieure de Paris, s'est déroulée du 4 juillet au 5 août 2016 sous la direction scientifique de Vincent Jolivet et sous la direction opérationnelle d'Edwige Lovergne, en étroite collaboration avec la Soprintendenza archeologica del Lazio e dell'Etruria meridionale (inspectrice: Dott.ssa Valeria D'Atri); le plan de sécurité a été rédigé par l'arch. Alessandro Bertollini, les travaux de chantier réalisés par l'entreprise Enzo Nicolamme de Monterazzano. Le financement a été assuré par l'UMR 8546 (AOrOc) du CNRS, le Département des Sciences de l'Antiquité dell'École normale supérieure de Paris, l'ANR CAECINA, le Labex TransferS (programme 
Investissements d'avenir ANR-10-IDEX-000102 PSL* et ANR-10-LABX-0099), et la Fondazione Carivit de Viterbe. Maria et Enzo Pepponi, propriétaires du site, ainsi que Felice Orlandini, inspecteur honoraire des Biens culturels, ont largement contribué, de multiples manières, à la réalisation de l'ensemble de l'opération.

Cette campagne a également permis de nouer de nouveaux rapports de collaboration : Stefano Urbini (Istituto nazionale di geofisica e vulcanologia, Rome) a réalisé des prospections Géoradar sur la tombe et à ses abords; Piero Ciccioli (CNR, Istituto di metodologie chimiche, Rome) et Cristina Di Salvo (Istituto di geologia ambientale $e$ geoingegneria) ont entrepris l'étude du contexte géologique de la tombe et des interventions de restauration possibles; Jean-Emmanuel Deschaud (École des Mines Paritech, Paris) et Frédérique Marchand-Beaulieu (CNRS, UMR 8546), ont réalisé la photogrammétrie et le scan 3D de la tombe; Benjamin Houal (Paris) a effectué par drone des reprises photographiques et vidéo de la tombe et de la vallée de la Leia, jusqu'à Musarna ; enfin, Marion Ellena (ENSAD, Paris), en collaboration avec Olivier Geli, a réalisé un film documentaire sur cette campagne de fouilles.

La seconde chambre funéraire de la tombe, creusée dans l'axe du dromos fouillé l'an dernier, s'est révélée entièrement obstruée de terre à la suite de l'effondrement de toute la partie centrale de son plafond, qu'il a fallu démonter depuis la surface pour pouvoir la fouiller. Creusée dans un tuf friable, elle présente un plan subrectangulaire, de 5,50 x $6 \mathrm{~m}$ de côté, pour une hauteur maximale de $1,90 \mathrm{~m}$, avec une amorce de cunicule, profond de $1,60 \mathrm{~m}$, dans sa paroi postérieure (fig. 1).

Fig. 1 - Vue zénithale de la seconde chambre funéraire, en cours de fouille.

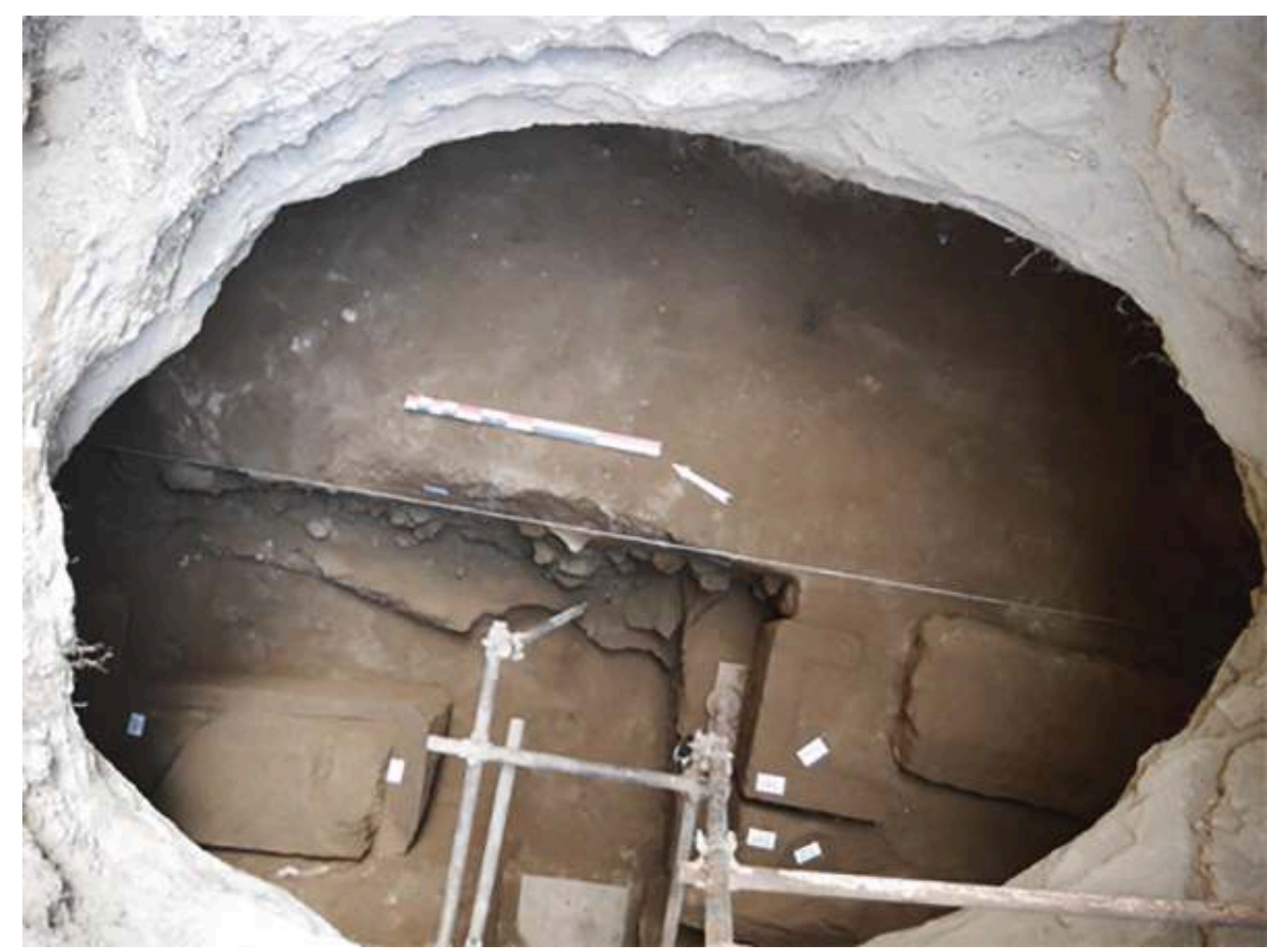

E. Lovergne.

Le côté droit est occupé par une banquette sur laquelle reposent trois sarcophages dont les couvercles ont été brisés et déplacés; deux d'entre eux portent une inscription sur 
leur côté court, teśi et cinial. Sur la gauche, un quatrième sarcophage de même type, également pillé, a été placé le long de la paroi ouest de la tombe. La plupart des objets composant le mobilier funéraire ont donc disparu, à l'exception d'un lot formé de deux grands unguentaria fusiformes, de quelques vases en céramique à vernis noir, ainsi que d'une fiaschetta et d'un miroir en bronze, retrouvés au sol près du sarcophage de droite ; à l'entrée du cunicule, au fond de la tombe, on a recueilli un ensemble de petits objets en bronze, dont trois pieds de ciste (fig. 2). L'ensemble des marqueurs de genre (miroir, ciste, fiaschetta, inscriptions, ainsi que des résidus de fard rose) semble confirmer que cette seconde chambre était réservée aux femmes.

Fig. 2 - Plan de la seconde chambre funéraire.

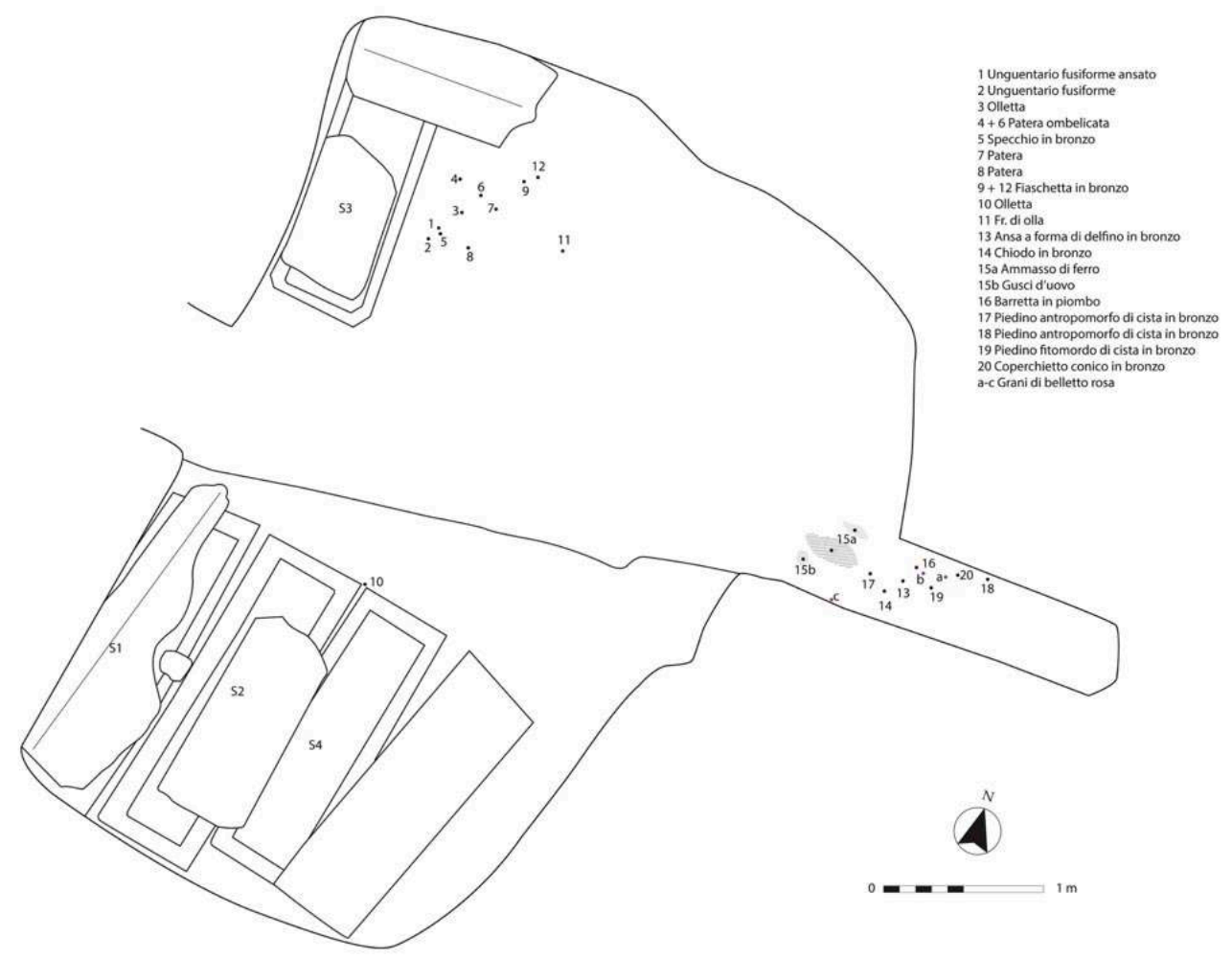

Relevé : A. Daumont-Marx, M. De Jonghe ; infographie : E. Lovergne.

La prospection Géoradar réalisée par S. Urbini à l'intérieur de l'hypogée principal a mis en évidence, au centre de la chambre, une anomalie d'environ $1 \times 2 \mathrm{~m}$ qui pourrait correspondre à un puits creusé pour atteindre un niveau de tuf plus compact, et creuser une chambre au-dessous de celle que nous connaissons. Si cette hypothèse est juste, l'accès à cette salle devait se faire par un escalier dont le départ serait dissimulé par le sarcophage placé dans la niche située à gauche de l'entrée.

Le long de la pente, une tranchée large de $1,80 \mathrm{~m}$, pour $22 \mathrm{~m}$ de longueur, implantée dans l'axe du dromos, a mis en évidence une épaisse couche de blocs de tuf sans doute en relation avec les travaux de dégagement du niveau d'effondrement de la façade du monument, qui semblent remonter au XVI siècle ; la fouille n'a pu être achevée cette année. Devant la tombe, au niveau de la rupture de pente, un mur monté en blocs de tuf grossièrement équarris, posé sur cette couche, appartenait probablement au soutènement de la voie qui donnait accès au monument à l'époque moderne. 
7 Enfin, la tranchée implantée sur le petit oppidum voisin de l'Isolotto, dont la fouille est dirigée par Pascal Neaud (INRAP, Reims), a permis de mettre au jour les fondations de cinq murs en grand appareil (fig. 3), qui confirment la présence d'une petite fortification construite sur le site au début de l'époque hellénistique.

Fig. 3 - Oppidum de l'Isolotto : les fondations en grand appareil.

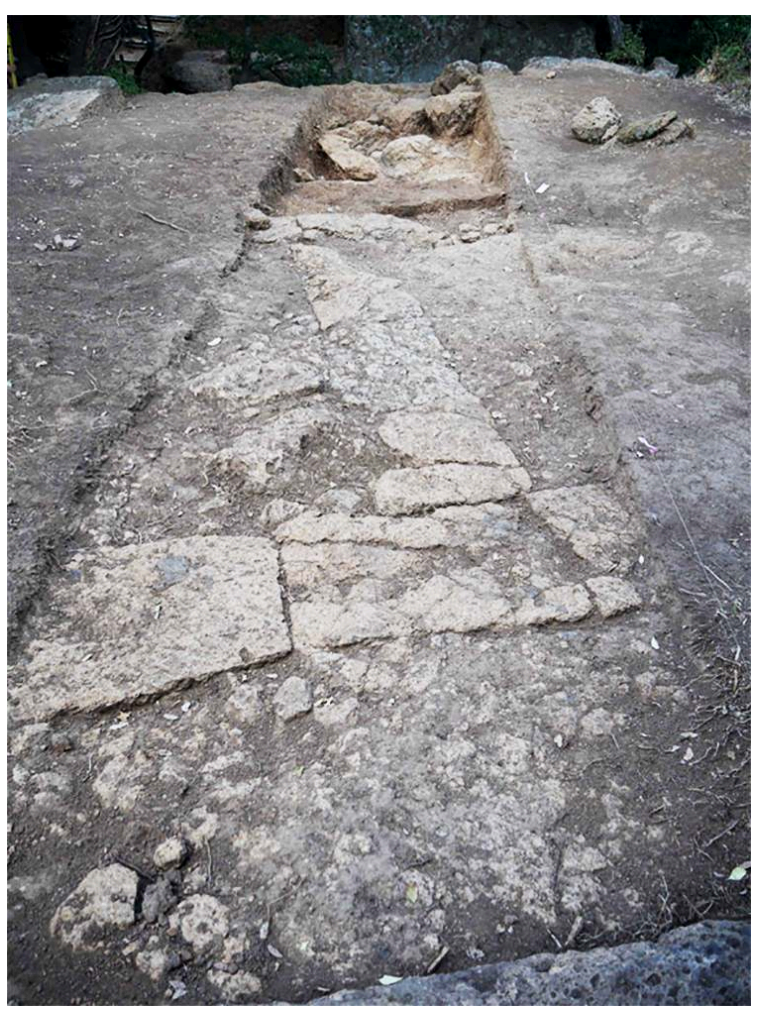

P. Neaud.

8 La prochaine campagne de fouilles devrait permettre d'améliorer les conditions de préservation et de présentation du monument au public, et de conclure l'ensemble des opérations engagées à ce jour : achèvement de la tranchée ouverte devant la tombe, fouille en extension des structures de l'Isolotto. Elle offrira également l'opportunité de vérifier s'il existe, ou non, une chambre funéraire plus ancienne, creusée sous celle que nous avons fouillée en 2014.

\section{INDEX}

institutions Soprintendenza archeologica del Lazio e dell'Etruria meridionale, AOROC (UMR 8546 CNRS), École normale supérieure (Paris) 
AUTEURS

VINCENT JOLIVET

CNRS, AOROC (UMR 8546 CNRS-ENS) - vincent.jolivet[at]ens.fr

EDWIGE LOVERGNE

UMR 7041 (ArScAn) - edwigelovergne[at]hotmail.com 